\title{
Maximal violation of a broad class of Bell inequalities and its implication on self-testing
}

\author{
C. Jebarathinam $\odot,{ }^{1}$ Jui-Chen Hung, ${ }^{2}$ Shin-Liang Chen $\odot,{ }^{1,3}$ and Yeong-Cherng Liang $\circledast^{1,4, *}$ \\ ${ }^{1}$ Department of Physics and Center for Quantum Frontiers of Research \& Technology (QFort), \\ National Cheng Kung University, Tainan 701, Taiwan \\ ${ }^{2}$ Department of Physics, National Cheng Kung University, Tainan 701, Taiwan \\ ${ }^{3}$ Dahlem Center for Complex Quantum Systems, Freie Universität Berlin, 14195 Berlin, Germany \\ ${ }^{4}$ Physics Division, National Center for Theoretical Sciences, Hsinchu 300, Taiwan
}

(Received 2 August 2019; published 4 November 2019)

\begin{abstract}
In quantum information, lifting is a systematic procedure that can be used to derive-when provided with a seed Bell inequality—other Bell inequalities applicable in more complicated Bell scenarios. It is known that the procedure of lifting introduced by Pironio [J. Math. Phys. 46, 062112 (2005)] preserves the facet-defining property of a Bell inequality. Lifted Bell inequalities therefore represent a broad class of Bell inequalities that can be found in all Bell scenarios. Here, we show that the maximal value of any lifted Bell inequality is preserved for both the set of nonsignaling correlations and quantum correlations. Despite the degeneracy in the maximizers of such inequalities, we show that the ability to self-test a quantum state is preserved under these lifting operations. In addition, except for outcome lifting, local measurements that are self-testable using the original Bell inequality—despite the degeneracy—can also be self-tested using any lifted Bell inequality derived therefrom. While it is not possible to self-test all the positive-operator-valued measure elements using an outcome-lifted Bell inequality, we show that partial, but robust self-testing statements on the underlying measurements can nonetheless be made from the quantum violation of these lifted inequalities. We also highlight the implication of our observations on the usefulness of using lifted Bell-like inequalities as a device-independent witnesses for entanglement depth. The impact of the aforementioned degeneracy on the geometry of the quantum set of correlations is briefly discussed.
\end{abstract}

DOI: 10.1103/PhysRevResearch.1.033073

\section{INTRODUCTION}

Inspired by the thought-provoking paper of Einstein, Podolsky, and Rosen [1], Bell derived [2]—-based on wellaccepted classical intuitions - an inequality constraining the correlations between local measurement outcomes on two distant systems. He further showed that the so-called Bell inequality can be violated by quantum theory using local but incompatible measurements on entangled states. Since then, various Bell inequalities, such as the one due to Clauser, Horne, Shimony and Holt (CHSH) [3], have been derived to investigate the intriguing nature of quantum theory and also the information-processing power enabled by these nonclassical, Bell-nonlocal [4] correlations.

For example, Ekert [5] showed in 1991 that the quantum violation of Bell inequalities offers an unprecedented level of security for quantum key distribution protocols. Independently, Mayers and Yao [6,7] showed that certain extremal quantum correlation enables the possibility to self-test quantum devices. These discoveries prompted the paradigm of deviceindependent quantum information $[4,8]$ in which the physical

\footnotetext{
*ycliang@mail.ncku.edu.tw

Published by the American Physical Society under the terms of the Creative Commons Attribution 4.0 International license. Further distribution of this work must maintain attribution to the author(s) and the published article's title, journal citation, and DOI.
}

properties of quantum devices are certified without making any assumption on the internal working of the devices, but rather through the observation of a Bell-nonlocal correlation.

Interestingly, an observation of the maximal quantum violation of certain Bell inequalities, such as the CHSH inequality, is already sufficient to self-test the underlying quantum state and the measurements that give rise to the observed violation [9]. To date, numerous Bell inequalities have been derived (see, e.g., [4,10-32] and references therein). However, beyond the CHSH Bell inequality, only a handful of them $[25,28,31,33-39]$ have been identified as relevant for the purpose of self-testing (see [40] for a recent review on the topic of self-testing). Is it possible to make some general statements regarding the self-testing property of Bell inequalities defined for an arbitrary Bell scenario?

To answer the above question, we consider, in this work, Bell inequalities that may be obtained from the procedure of Pironio's lifting [41] (see Fig. 1). Importantly, such inequalities exist in all Bell scenarios beyond the simplest one for two parties, two settings, and two outcomes. If a Bell inequality is facet defining [16], the same holds for its liftings [41]. What about their quantum violation? In [42], it was shown that in addition to the bound satisfied by Bell-local correlations, both the maximal quantum value and the maximal nonsignaling [43] value of Bell inequalities are preserved for party lifting. In this work, we give an alternative proof of this fact and show, in addition, that both the maximal quantum value and the maximal nonsignaling value of a Bell inequality are also preserved for other types of Pironio's lifting. 


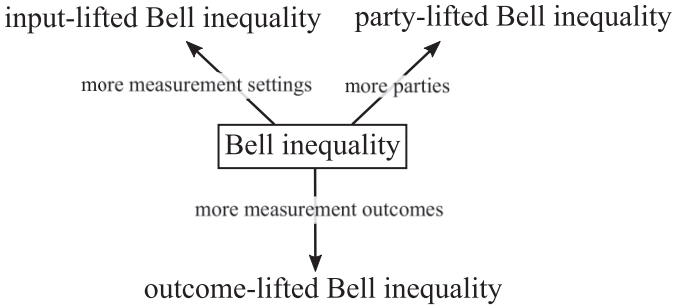

FIG. 1. Summary of the three distinct types of liftings [41] considered in this work. Upon the application of lifting, a Bell inequality becomes applicable in a more complicated Bell scenario, such as one with more measurement settings, or one with more measurement outcomes for at least one of the settings, or one with more parties. Of course, concatenation of distinct types of liftings are also possible.

As a corollary of our results, we further show that the self-testing properties of a Bell inequality is largely preserved through the procedure of lifting. In other words, if a Bell inequality can be used to self-test some quantum state $|\psi\rangle$, so can its liftings. Moreover, except for the case of outcome lifting, the possibility to self-test the underlying positiveoperator-valued measure (POVM) using a Bell inequality remains intact upon the application of Pironio's lifting. As we illustrate in this work, the maximizers of lifted Bell inequalities are not unique. There is thus no hope (see, e.g., [44]) of providing a complete self-testing of the employed quantum devices using a lifted Bell inequality. Nonetheless, we provide numerical evidence suggesting that lifted Bell inequalities provide the same level of robustness for selftesting the relevant parts of the devices.

The rest of this paper is organized as follows. In Sec. II, we introduce basic notions of a Bell scenario and recall the definitions of self-testing. After that, we investigate and compare the maximal violation of lifted Bell inequalities against that of the original Bell inequalities, assuming quantum, or general nonsignaling, correlations [43]. In the same section, we also discuss the self-testing property of lifted Bell inequalities, and the usefulness of party-lifted Bell-like inequalities as device-independent witnesses for entanglement depth [26]. In Sec. IV, we present some concluding remarks and possibilities for future research. Examples illustrating the nonuniqueness of the maximizers of lifted Bell inequalities, as well as their implications on the geometry of the quantum set of correlations are provided in the Appendixes.

\section{PRELIMINARIES}

\section{A. Bell scenario}

Consider a Bell scenario involving $n$ spatially separated parties labeled by $i \in\{1,2, \ldots, n\}$ and let the $i$ th party perform a measurement labeled by $j_{i}$,with outcomes labeled by $k_{i}$. In such a setup, we may appreciate the strength of correlation between the observed measurement outcomes via a collection of joint conditional probabilities. Following the literature (see, e.g., [4]), we represent these conditional probabilities-dubbed a correlation-of observing the outcome combination $\vec{k}=\left(k_{1} k_{2} \ldots k_{n}\right)$ conditioned on

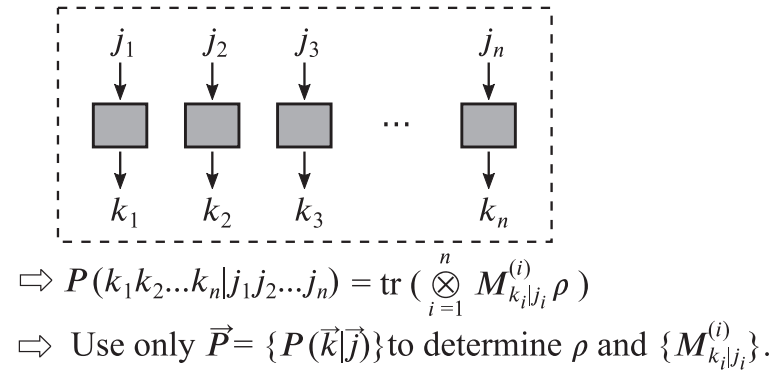

FIG. 2. In a Bell test, the raw data collected can be used to estimate the underlying conditional probability distribution $P(\vec{k} \mid \vec{j})$, which manifests the extent to which the measurement outcomes are correlated (see, however, Ref. [45] for subtleties involved in such an estimation). If this correlation is strong enough, it may be used for device-independent characterizations, i.e., the task of deducing, directly from $\vec{P}=\{P(\vec{k} \mid \vec{j})\}$ and Born's rule, certain properties of the shared state $\rho$ and the local POVM $\left\{M_{k_{i} \mid j_{i}}^{(i)}\right\}$. The strongest form of such characterizations is known as self-testing, which is to deducemodulo unimportant local degrees of freedom-exactly what $\rho$ and $\left\{M_{k_{i} \mid j_{i}}^{(i)}\right\}$ are.

performing the measurements $\vec{j}=\left(j_{1} j_{2} \ldots j_{n}\right)$ by the vector $\vec{P}:=\{P(\vec{k} \mid \vec{j})\}$ (see Fig. 2).

In addition to the normalization condition and the positivity constraint $P(\vec{k} \mid \vec{j}) \geqslant 0$ for all $\vec{k}, \vec{j}$, each correlation is required to satisfy the nonsignaling constraints [43] (see also [46])

$$
\begin{aligned}
& \sum_{k_{i}} P\left(k_{1} \ldots k_{i} \ldots k_{n} \mid j_{1} \ldots j_{i} \ldots j_{n}\right) \\
& \quad=\sum_{k_{i}} P\left(k_{1} \ldots k_{i} \ldots k_{n} \mid j_{1} \ldots j_{i}^{\prime} \ldots j_{n}\right)
\end{aligned}
$$

for all $i, j_{1}, \ldots j_{i-1}, j_{i}, j_{i}^{\prime}, j_{i+1} \ldots j_{n}$, and $k_{\ell}$ (with $\ell \neq i$ ). In any given Bell scenario, the set of correlations satisfying these constraints forms the so-called nonsignaling polytope $\mathcal{N}$ [43].

A correlation is called Bell local [4] if it can be explained by a local-hidden-variable model [2],

$$
P\left(k_{1} \ldots k_{n} \mid j_{1} \ldots j_{n}\right)=\sum_{\lambda} p_{\lambda} P\left(k_{1} \mid j_{1}, \lambda\right) \ldots P\left(k_{n} \mid j_{n}, \lambda\right)
$$

for all $k_{1} \ldots k_{n}, j_{1} \ldots j_{n}$, where $\lambda$ is the hidden variable which occurs with probability $p_{\lambda}, \sum_{\lambda} p_{\lambda}=1$ and $P\left(k_{i} \mid j_{i}, \lambda\right)$ is the probability of obtaining the measurement outcome $k_{i}$ given the setting $j_{i}$ and the hidden variable $\lambda$. As with the nonsignaling polytope $\mathcal{N}$, the set of Bell-local correlations forms a polytope (often called a local polytope $\mathcal{L}$ ), which is a subset of $\mathcal{N}$

A correlation which cannot be explained by a local-hiddenvariable model is said to be Bell nonlocal and must necessarily violate a Bell inequality [2]—a constraint satisfied by all $\vec{P} \in$ $\mathcal{L}$. A linear Bell inequality has the generic form

$$
I_{n}(\vec{P}):=\vec{B} \cdot \vec{P}=\sum_{k_{1} \cdots k_{n}, j_{1} \cdots j_{n}} B_{\vec{k}, \vec{j}} P(\vec{k} \mid \vec{j}) \stackrel{\mathcal{L}}{\leqslant} \beta_{\mathcal{L}},
$$

where $\vec{B}:=\left\{B_{\vec{k}, \vec{j}}\right\}$ denotes the vector of Bell coefficients, and $\vec{B} \cdot \vec{P}$ and $\beta_{\mathcal{L}}$ are, respectively, the Bell expression and the local bound of a Bell inequality. 


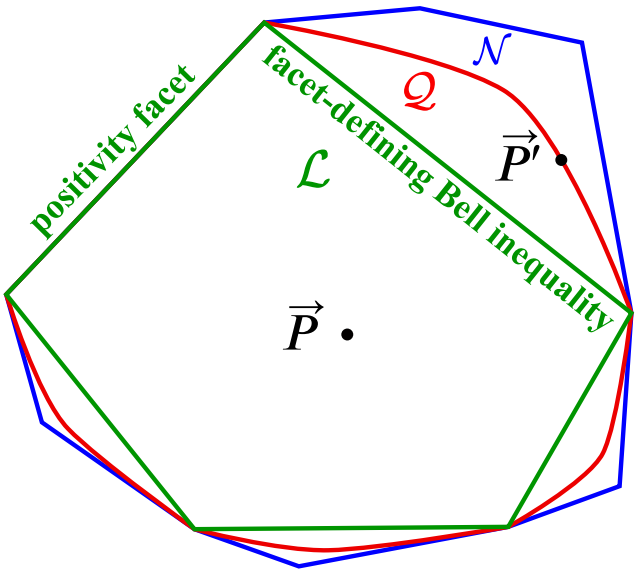

FIG. 3. Schematic illustrating the space of all correlations $\vec{P}$, as well as the inclusion relations between the set of Bell-local correlations $\mathcal{L}$ (innermost, green boundary), the set of quantum correlations $\mathcal{Q}$ (red boundary), and the set of nonsignaling correlations $\mathcal{N}$ (outermost, blue boundary). Note that a positivity facet (which dictates that a legitimate probability is non-negative) represents a boundary that is (partially) shared by all three sets while a nontrivial (facet-defining) Bell inequality allows one to distinguish a non-Bell-local correlation, such as $\vec{P}^{\prime}$, from those $\vec{P} \in \mathcal{L}$. Note that to perform self-testing, such a $\vec{P}^{\prime}$ must lie on the boundary of $\mathcal{Q}$ (see [44]).

A correlation $\vec{P}$ is called quantum if the joint probabilities can be written as

$$
P\left(k_{1} \ldots k_{n} \mid j_{1} \ldots j_{n}\right)=\operatorname{tr}\left(\otimes_{i=1}^{n} M_{k_{i} \mid j_{i}}^{(i)} \rho_{12 \ldots n}\right),
$$

where $\rho_{12 \ldots n}$ is an $n$-partite density matrix and $\left\{M_{k_{i} \mid j_{i}}^{(i)}\right\}_{k_{i}}$ is the POVM describing the $j_{i}$ th measurement of the $i$ party. By definition, POVM elements satisfy the constraints of being positive semidefinite, $M_{k_{i} \mid j_{i}}^{(i)} \geq 0$ for all $k_{i}$ and $j_{i}$, as well as the normalization requirement $\sum_{k_{i}} M_{k_{i} \mid j_{i}}^{(i)}=\mathbf{1}$ for all $j_{i}$. Thus, a correlation is quantum if and only if the joint probabilities of such a correlation can be realized experimentally by performing local measurements on an $n$-partite quantum system. The set of quantum correlations $\mathcal{Q}$ forms a convex set satisfying $\mathcal{L} \subset \mathcal{Q} \subset \mathcal{N}$ (see Fig. 3). It is, however, not a polytope [11] (see also [44]). When necessary, we will use $\mathcal{Q}_{n}$ to denote the set of quantum correlations arising in an $n$-partite Bell scenario.

\section{B. Self-testing}

Certain nonlocal correlations $\vec{P} \in \mathcal{Q}$ have the appealing feature of being able to reveal (essentially unambiguously) the quantum strategy, i.e., the underlying state and/or the POVM leading to these correlations $[7,9,11,40]$. Following [7], we say that such a $\vec{P} \in \mathcal{Q}$ self-tests the underlying quantum strategy. To this end, it is worth noting that all pure bipartite entangled states can be self-tested [47].

To facilitate subsequent discussions, we recall from [44] the formal definition of self-testing in a bipartite Bell scenario (see also [40]). Specifically, consider two spatially separated parties Alice and Bob who each performs measurements labeled by $x, y$ and, respectively, observes the outcomes $a, b$.
We say that a bipartite correlation $\vec{P}:=\{P(a b \mid x y)\}$ satisfying

$$
P(a b \mid x y)=\operatorname{tr}\left[M_{a \mid x}^{(1)} \otimes M_{b \mid y}^{(2)} \rho_{12}\right]
$$

for all $a, b, x$, and $y$ self-tests the reference (entangled) state $\left|\tilde{\psi}_{12}\right\rangle$ if there exists a local isometry $\Phi=\Phi_{1} \otimes \Phi_{2}$ such that

$$
\Phi \rho_{12} \Phi^{\dagger}=\left|\tilde{\psi}_{12}\right\rangle\left\langle\tilde{\psi}_{12}\right| \otimes \rho_{\text {aux }}
$$

where $\rho_{12}$ is the measured quantum state [acting on $\mathcal{H}_{A} \otimes$ $\left.\mathcal{H}_{B}\right], \rho_{\text {aux }}$ is an auxiliary state acting on $\mathcal{H}_{A^{\prime}} \otimes \mathcal{H}_{B^{\prime}}$, and $\mathcal{H}_{A^{\prime}}$ and $\mathcal{H}_{B^{\prime}}$ are the Hilbert spaces associated with the other degrees of freedom of Alice and Bob's subsystem respectively [48].

Often, a $\vec{P} \in \mathcal{Q}$ that self-tests some reference quantum state can also be used to certify the measurements as well. In such cases, we say that a bipartite correlation $\vec{P}$ obtained from Eq. (4) self-tests the reference quantum state $\left|\tilde{\psi}_{12}\right\rangle$ and the reference POVM $\left\{\tilde{M}_{a \mid x}^{(1)}\right\}_{a},\left\{\tilde{M}_{b \mid y}^{(2)}\right\}_{b}$ if there exists a local isometry $\Phi=\Phi_{1} \otimes \Phi_{2}$ such that Eq. (5) holds and

$$
\Phi\left[M_{a \mid x}^{(1)} \otimes M_{b \mid y}^{(2)} \rho_{12}\right] \Phi^{\dagger}=\left[\tilde{M}_{a \mid x}^{(1)} \otimes \tilde{M}_{b \mid y}^{(2)}\left|\tilde{\psi}_{12}\right\rangle\left\langle\tilde{\psi}_{12}\right|\right] \otimes \rho_{\text {aux }} .
$$

for all $a, b, x$, and $y$. By summing over $a, b$, and using the normalization of POVM, one recovers Eq. (5) from Eq. (6).

Interestingly, there are Bell inequalities whose maximal quantum violation alone is sufficient to self-test the quantum state (and the POVM) [28,34,36,37,49-52]. Since then, identifying Bell inequalities which can be used for the task of self-testing has received considerable attention. To this end, note that if the maximal quantum violation of a Bell inequality self-tests some quantum state as well as the underlying measurements, then this maximal quantum violation must be achieved by a unique $\vec{P} \in \mathcal{Q}$ [44].

More formally, consider a bipartite Bell inequality,

$$
I_{2}(\vec{P}):=\sum_{a, b, x, y} B_{a b x y} P(a b \mid x y) \stackrel{\mathcal{L}}{\leqslant} \beta_{\mathcal{L}},
$$

with a quantum bound (maximal quantum violation):

$$
\beta_{\mathcal{Q}}=\max _{\vec{P} \in \mathcal{Q}} I_{2}(\vec{P})>\beta_{\mathcal{L}} .
$$

We say that an observation of the quantum violation $I_{2}(\vec{P})=$ $\beta_{\mathcal{Q}}$ self-tests the reference (entangled) state $\left|\tilde{\psi}_{12}\right\rangle$ and the reference POVM $\left\{\tilde{M}_{a \mid x}^{(1)}\right\}_{a},\left\{\tilde{M}_{b \mid y}^{(2)}\right\}_{b}$ if there exists a local isometry $\Phi=\Phi_{1} \otimes \Phi_{2}$ such that Eq. (6) holds for all $\vec{P} \in \mathcal{Q}$ [cf. Eq. (4)] satisfying $I_{2}(\vec{P})=\beta_{\mathcal{Q}}$.

\section{MAXIMAL VIOLATION OF LIFTED BELL INEQUALITIES AND ITS IMPLICATIONS}

In this section, we show that both the maximal quantum violation and the nonsignaling violation of a lifted Bell inequality must be the same as that of the Bell inequality from which the lifting is applied. We then discuss the implication of these observations in the context of self-testing (Fig. 2), on the geometry of the quantum set of correlations (Fig. 3), as well as on the device-independent certification of entanglement depth. For ease of presentation, our discussion will often be carried out assuming a bipartite Bell scenario (for the original 
Bell inequality). However, it should be obvious from the presentation that our results also hold for any Bell scenario with more parties, and also for a Bell inequality that is not necessarily facet defining.

\section{A. More inputs}

Let us begin with the simplest kind of lifting, namely, one that allows additional measurement settings. Applying Pironio's input lifting [41] to a Bell inequality means to consider the very same Bell inequality in a Bell scenario with more measurement settings for at least one of the parties. At first glance, it may seem rather unusual to make use of only the data collected for a subset of the input combinations, but in certain cases (see, e.g., [53]), the consideration of all inputlifted facets is already sufficient to identify the non-Bell-local nature of the observed correlations.

Since an input-lifted Bell inequality is exactly the same as the original Bell inequality, its maximal quantum and nonsignaling violation is obviously the same as that of the original Bell inequality. Similarly, it is evident that if the maximal quantum violation of the original Bell inequality self-tests some reference quantum state $|\psi\rangle$ and POVMs $\left\{M_{a \mid x}^{(1)}\right\}_{a, x},\left\{M_{b \mid y}^{(2)}\right\}_{b, y}, \ldots$, so does the maximal quantum violation of the input-lifted Bell inequality.

However, since no constraint is imposed on the additional inputs that do not appear in the Bell expression, it is clear that even if we impose the constraint that the maximal quantum violation of an input-lifted Bell inequality is attained, these other local POVMs can be completely arbitrary. Thus, the subset of quantum correlation attaining the maximal quantum violation of any input-lifted Bell inequality is not unique, and has a degeneracy that increases with the number of these "free" inputs. In other words, the set of quantum maximizers of any input-lifted Bell inequality define a flat region of the boundary of the quantum set of correlations, cf. [44]. In particular, it could lead to completely flat boundaries of $\mathcal{Q}$ on specific two-dimensional slices in the correlation space (see Fig. 4). For some explicit examples illustrating the aforementioned nonuniqueness, see Appendix A.

\section{B. More outcomes}

Instead of the trivial input lifting, one may also lift a Bell inequality to a scenario with more measurement outcomes. Specifically, consider a bipartite Bell scenario where the $y^{\prime}$ th measurement of Bob has $v \geqslant 2$ possible outcomes. The simplest outcome lifting à la Pironio [41] then consists of two steps: (1) choose an outcome, say, $b=b^{\prime}$ from Bob's $y^{\prime}$ th measurement, and (2) replaces in the sum of Eq. (7) all terms of the form $P\left(a b^{\prime} \mid x y^{\prime}\right)$ by $P\left(a b^{\prime} \mid x y^{\prime}\right)+P\left(a u \mid x y^{\prime}\right)$.

The resulting outcome-lifted Bell inequality reads as

$$
\begin{aligned}
I_{2}^{\mathrm{LO}}= & \sum_{a, b, x, y \neq y^{\prime}} B_{a b x y} P(a b \mid x y)+\sum_{a, x, b \neq b^{\prime}, u} B_{a b x y^{\prime}} P\left(a b \mid x y^{\prime}\right) \\
& +\sum_{a, x} B_{a b^{\prime} x y^{\prime}}\left[P\left(a b^{\prime} \mid x y^{\prime}\right)+P\left(a u \mid x y^{\prime}\right)\right] \stackrel{\mathcal{L}}{\leqslant} \beta_{L},
\end{aligned}
$$

where the local bound $\beta_{\mathcal{L}}$ is provably the same [41] as that of the original Bell inequality, Eq. (7). It is worth noting that

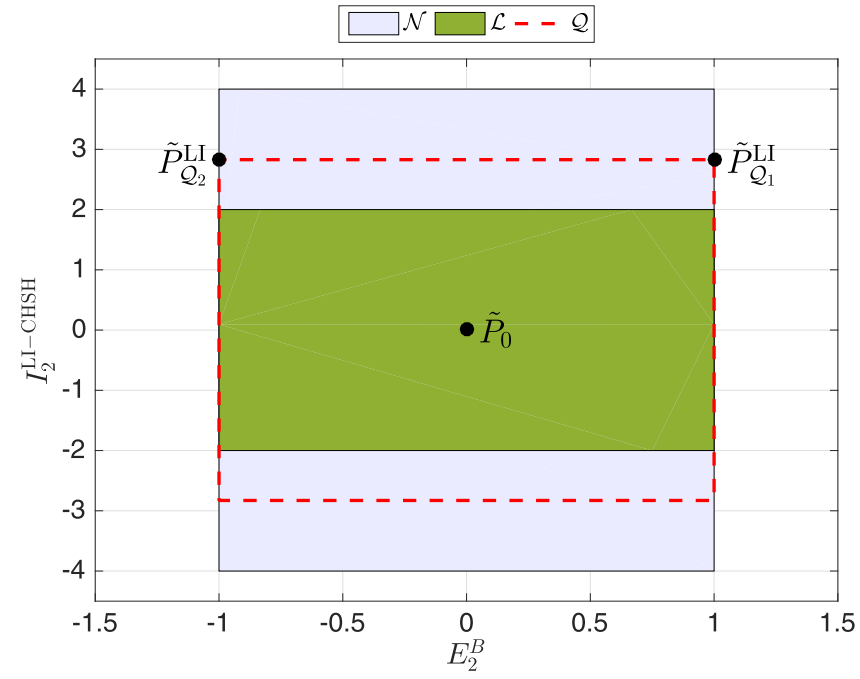

FIG. 4. A two-dimensional slice in the input-lifted space of correlations spanned by $\tilde{P}_{Q_{1}}^{\mathrm{LO}}, \tilde{P}_{Q_{2}}^{\mathrm{LO}}$ [see Eq. (A5)] and the uniform distribution $\tilde{P}_{0}$. From the innermost to the outermost, we have, respectively, the set of Bell-local correlations $\mathcal{L}$ (green), the set of quantum correlations $\mathcal{Q}$ (red, dashed boundary), and the set of nonsignaling correlations $\mathcal{N}$ (mauve). To illustrate the degeneracy in the maximally violating correlations, we have chosen the input-lifted Bell inequality of Eq. (A3) and the marginal correlator for $y=2$, i.e., $E_{2}^{B}=\tilde{P}(b=0 \mid y=2)-\tilde{P}(b=1 \mid y=2)$ as, respectively, the vertical and horizontal axis of this plot. As opposed to the two-dimensional slices shown in [44], the set of quantum correlations $\mathcal{Q}$ appears to be a rectangle on this slice.

outcome-lifted Bell inequalities arise naturally in the study of detection loopholes in Bell experiments; see, e.g., [30,54].

\section{Preservation of quantum and nonsignaling violation}

As with input lifting, we now proceed to demonstrate the invariance of maximal Bell violation with outcome lifting.

Proposition 1. Lifting of outcomes preserves the quantum bound and the nonsignaling bound of any Bell inequality, i.e., $\beta_{\mathcal{Q}}^{\mathrm{LO}}=\beta_{\mathcal{Q}}$ and $\beta_{\mathcal{N}}^{\mathrm{LO}}=\beta_{\mathcal{N}}$, where $\beta_{\mathcal{Q}}\left(\beta_{\mathcal{Q}}^{\mathrm{LO}}\right)$ and $\beta_{\mathcal{N}}\left(\beta_{\mathcal{N}}^{\mathrm{LO}}\right)$ are, respectively, the quantum and the nonsignaling bounds of the original (outcome-lifted) Bell inequality.

Proof. From Eq. (9), one clearly sees that the $b^{\prime}$ th outcome and the $u$ th outcome of Bob's $y^{\prime}$ th measurement are treated on equal footing. So, we may as well consider Bob's $y^{\prime}$ th measurement as an effective $v$-outcome measurement by considering its $b^{\prime}$ th outcome and its $u$ th outcome together as one outcome. Hence, if we define

$$
\begin{gathered}
\tilde{P}(a b \mid x y)=P(a b \mid x y), \quad y \neq y^{\prime}, \\
\tilde{P}\left(a b \mid x y^{\prime}\right)=P\left(a b \mid x y^{\prime}\right), \quad b \notin\left\{b^{\prime}, u\right\}, \\
\tilde{P}\left(a b^{\prime} \mid x y^{\prime}\right)=P\left(a b^{\prime} \mid x y^{\prime}\right)+P\left(a u \mid x y^{\prime}\right)
\end{gathered}
$$

and substitute it back into Eq. (9), we recover the Bell expression of the original Bell inequality [left-hand side of Eq. (7)] by identifying $\tilde{P}(a b \mid x y)$ in $I_{2}^{\mathrm{LO}}$ as $P(a b \mid x y)$ in $I_{2}$. Moreover, if $\vec{P}$ defined for this more-outcome Bell scenario 
is quantum (nonsignaling), the resulting correlation obtained with the coarse-graining procedure of Eq. (10) is still quantum (nonsignaling). A proof of this for quantum correlations is provided in Appendix B (see, e.g., [55] for the case of nonsignaling correlation).

This implies that for any violation of the outcome-lifted Bell inequality (9) by a quantum (or a nonsignaling) correlation, there always exists another quantum (respectively, a nonsignaling) correlation that gives the same amount of violation for the original Bell inequality (7). In particular, the maximal quantum and nonsignaling violation of these inequalities must satisfy

$$
\beta_{\mathcal{N}} \geqslant \beta_{\mathcal{N}}^{\mathrm{LO}} \quad \beta_{\mathcal{Q}} \geqslant \beta_{\mathcal{Q}}^{\mathrm{LO}} .
$$

On the other hand, instead of grouping the outcomes in the outcome-lifted Bell scenario, one could also start from the original Bell scenario and (arbitrarily) split the $b^{\prime}$ th outcome of Bob's $y^{\prime}$ th measurement into two outcomes labeled by $b=$ $b^{\prime}$ and $b=u$. Hence, if we define $\widehat{P}\left(a b^{\prime} \mid x y^{\prime}\right), \widehat{P}\left(a u \mid x y^{\prime}\right)$ in the outcome-lifted Bell scenario such that

$$
\begin{aligned}
\widehat{P}(a b \mid x y)= & P(a b \mid x y), \quad y \neq y^{\prime} \quad \text { or } y=y^{\prime}, \quad b \neq b^{\prime}, u, \\
0 \leqslant & \widehat{P}\left(a b^{\prime} \mid x y^{\prime}\right), \quad \widehat{P}\left(a u \mid x y^{\prime}\right) \leqslant 1, \\
& \widehat{P}\left(a b^{\prime} \mid x y^{\prime}\right)+\widehat{P}\left(a u \mid x y^{\prime}\right)=P\left(a b^{\prime} \mid x y^{\prime}\right)
\end{aligned}
$$

and substitute it into Eq. (7), we recover the outcomelifted Bell expression [Eq. (9)] by identifying $\widehat{P}\left(a b^{\prime} \mid x y^{\prime}\right)$ and $\widehat{P}\left(a u \mid x y^{\prime}\right)$, respectively, as $P\left(a b^{\prime} \mid x y^{\prime}\right)$ and $P\left(a u \mid x y^{\prime}\right)$ in $I_{2}^{\mathrm{LO}}$, cf Eq. (9).

Moreover, the correlation obtained by locally splitting the outcomes, as required in Eq. (12), is realizable quantum mechanically (see Appendix B) or in general nonsignaling theory (see [55]) if the original correlations are, respectively, quantum and nonsignaling. Hence, for any violation of the original Bell inequality [Eq. (7)] by a quantum (nonsignaling) correlation, there always exists a quantum (nonsignaling) correlation giving the same amount of violation for the outcomelifted Bell inequality (9), i.e.,

$$
\beta_{\mathcal{Q}} \leqslant \beta_{\mathcal{Q}}^{\mathrm{LO}} ; \quad \beta_{\mathcal{N}} \leqslant \beta_{\mathcal{N}}^{\mathrm{LO}} .
$$

Combining Eqs. (11) and (13), it then follows that the maximal quantum and nonsignaling violation of any Bell inequality is preserved through the procedure of outcome-lifting, i.e.,

$$
\beta_{\mathcal{Q}}=\beta_{\mathcal{Q}}^{\mathrm{LO}} ; \quad \beta_{\mathcal{N}}=\beta_{\mathcal{N}}^{\mathrm{LO}}
$$

This completes the proof when only one of the outcomes $(b=$ $u$ ) of one of the measurements $\left(y=y^{\prime}\right)$ of one of the parties (Bob) is lifted. However, since more complicated outcome lifting can be achieved by concatenating the simplest outcome lifting presented above, the proof for the general scenarios can also be obtained by concatenating the proof given above, thus completing the proof for the general scenario.

\section{Implications on self-testing}

As an implication of the above proposition, we obtain the following result in the context of quantum theory.

Corollary 1. If the maximal quantum violation of a Bell inequality self-tests a quantum state $|\tilde{\psi}\rangle$, then any
Bell inequality obtained therefrom by outcome lifting also self-tests $|\tilde{\psi}\rangle$.

Proof. For definiteness, we prove this for the specific case of $n=2$; the general proof is completely analogous. To this end, let $\rho_{12}^{*}$ denote an optimal quantum state that maximally violates the outcome-lifted Bell inequality (9) [which has $(v+1)$ outcomes for Bob's $y^{\prime}$ th measurement] with appropriate choice of POVMs, $\left\{M_{a \mid x}^{(1)}\right\}_{a, x},\left\{M_{b \mid y}^{(2)}\right\}_{b, y}$. As shown in Appendix B, this quantum state $\rho_{12}^{*}$ can also be used to realize an effective $v$-outcome distribution for Bob's $y^{\prime}$ th measurement by combining Bob's relevant POVM elements for this measurement into a single POVM element, thereby implementing the local coarse graining given in Eq. (10) to give the maximal quantum violation of the original Bell inequality, Eq. (7). Suppose that the maximal quantum violation of inequality (9) does not self-test the reference state $\left|\tilde{\psi}_{12}\right\rangle$, i.e., there does not exist any local isometry $\Phi=\Phi_{1} \otimes \Phi_{2}$ such that

$$
\Phi \rho_{12}^{*} \Phi^{\dagger}=\left|\tilde{\psi}_{12}\right\rangle\left\langle\tilde{\psi}_{12}\right| \otimes \rho_{\text {aux }}
$$

for some $\rho_{\text {aux }}$. Then, we see that the maximal quantum violation of inequality (7) (attainable using $\rho_{12}^{*}$ ) also cannot selftest the reference state $\left|\tilde{\psi}_{12}\right\rangle$. The desired conclusion follows by taking the contrapositive of the above implication.

A few remarks are now in order. As with any other Bell inequality, in examining the quantum violation of an outcome-lifted Bell inequality, one may consider arbitrary local POVMs having the right number of outcomes (acting on some given Hilbert space). A priori, they do not have to be related to the optimal POVM of the original Bell inequality. However, from the proof of Proposition 1, one notices that POVMs arising from splitting the outcomes of the original optimal POVM do play an important role in attaining the maximal quantum violation of the outcome-lifted Bell inequality.

The arbitrariness in this splitting, nonetheless, implies that $\vec{P} \in \mathcal{Q}$ maximally violating an outcome-lifted Bell inequality is not unique (see Appendix A 2 for some explicit examples). Since this invalidates a necessary requirement to self-test both the state and all the local POVMs (see Proposition C.1. of Ref. [44]), we must thus conclude-given that such an inequality preserves the ability to self-test the underlying state-that its maximal violation cannot be used to completely self-test the underlying measurements. Using the swap method of [56], we nevertheless show in Appendix $\mathrm{C}$ that the quantum violation of an outcome-lifted Bell inequality may still provide robust self-testing of some of the underlying POVM elements, as well as the nature of the merged POVM elements.

\section{More parties}

Finally, let us consider the party lifting of [41]. Again, for simplicity, we provide hereafter explicit constructions and proofs only for the bipartite scenario, with the multipartite generalizations proceeding analogously. To this end, it is expedient to write a generic bipartite Bell inequality such that

$$
I_{2}:=\sum_{a, b, x, y} B_{a b x y} P(a b \mid x y) \stackrel{\mathcal{L}}{\leqslant} 0,
$$


i.e., with its local bound set to zero. ${ }^{1}$ For any fixed but arbitrary input-output pair $c^{\prime}, z^{\prime}$ of the additional party (Charlie), applying the party lifting of [41] to inequality (16) gives rise to the tripartite Bell inequality:

$$
I_{2}^{\mathrm{LP}}:=\sum_{a, b, x, y} B_{a b x y} P\left(a b c^{\prime} \mid x y z^{\prime}\right) \stackrel{\mathcal{L}}{\leqslant} 0 .
$$

It is worth noting that such Bell inequalities have found applications in the foundations of quantum theory [57,58], as well as in the systematic generation [59] of device-independent witnesses for entanglement depth [26].

\section{Preservation of quantum and nonsignaling violation}

That the maximal quantum and nonsignaling violation remain unchanged under Pironio's party-lifting operation [41] follows directly from the results shown in Sec. 2.4 of [42]. For the convenience of subsequent discussions, however, we provide below an alternative proof of this observation.

Observation 1. Lifting of parties preserves the quantum bound and the nonsignaling bound of any Bell inequality, i.e., $\beta_{\mathcal{Q}}^{\mathrm{LP}}=\beta_{\mathcal{Q}}$ and $\beta_{\mathcal{N}}^{\mathrm{LP}}=\beta_{\mathcal{N}}$, where $\beta_{\mathcal{Q}}\left(\beta_{\mathcal{Q}}^{\mathrm{LP}}\right)$ and $\beta_{\mathcal{N}}\left(\beta_{\mathcal{N}}^{\mathrm{LP}}\right)$ are, respectively, the quantum and the nonsignaling bounds of the original (party-lifted) Bell inequality.

Proof. For a tripartite Bell scenario relevant to inequality (17), the marginal probability of Charlie getting the outcome $c^{\prime}$ conditioned on him performing the measurement labeled by $z^{\prime}$ is

$$
P\left(c^{\prime} \mid z^{\prime}\right)=\sum_{a, b} P\left(a b c^{\prime} \mid x y z^{\prime}\right) .
$$

Since the party-lifted inequality of Eq. (17) is saturated with the choice of $P\left(c^{\prime} \mid z^{\prime}\right)=0$, thereby making $P\left(a b c^{\prime} \mid x y z^{\prime}\right)=0$ for all $a, b, x, y$, the observation holds trivially if inequality (17) cannot be violated by general nonsignaling correlations.

Conversely, if inequality (17) can be violated by some quantum or general nonsignaling correlation, the corresponding $P\left(c^{\prime} \mid z^{\prime}\right)$ must be nonvanishing. Hence we thus assume that $P\left(c^{\prime} \mid z^{\prime}\right)>0$. To this end, note that

$$
P_{c^{\prime} \mid z^{\prime}}(a b \mid x y):=P\left(a b c^{\prime} \mid x y z^{\prime}\right) / P\left(c^{\prime} \mid z^{\prime}\right)
$$

gives the probabilities of Alice and Bob obtaining the outcomes $a$ and $b$ conditioned on her (him) choosing measurement $x(y)$, Charlie measuring $z^{\prime}$, and obtaining the outcome $c^{\prime}$. Note that the vector of probabilities $\vec{P}_{c^{\prime} \mid z^{\prime}}:=\left\{P_{c^{\prime} \mid z^{\prime}}(a b \mid x y)\right\}$ is a legitimate correlation in the (original) Bell scenario corresponding to inequality (16).

To prove the observation, we now focus on the case of finding the quantum bound, i.e., the maximum value of the left-hand side of Eq. (17) for quantum correlations - the proof for the nonsignaling case is completely analogous. To this end, note that the quantum bound of inequality (17) - given the above remarks—-satisfies

$$
\begin{aligned}
\beta_{\mathcal{Q}}^{\mathrm{LP}} & =\max _{\left\{P\left(a b c^{\prime} \mid x y z^{\prime}\right)\right\} \in \mathcal{Q}_{3}} \sum_{a, b, x, y} B_{a b x y} P\left(a b c^{\prime} \mid x y z^{\prime}\right) \\
& =\max _{\left\{P\left(a b c^{\prime} \mid x y z^{\prime}\right)\right\} \in \mathcal{Q}_{3}} \sum_{a, b, x, y} B_{a b x y} P_{c^{\prime} \mid z^{\prime}}(a b \mid x y) P\left(c^{\prime} \mid z^{\prime}\right)
\end{aligned}
$$

\footnotetext{
${ }^{1}$ This can always be achieved by (repeatedly) applying the identity of the form given in Eq. (1) to both sides of Eq. (7).
}

$$
\begin{aligned}
& \leqslant \max _{\vec{P}_{c^{\prime} \mid z^{\prime}} \in \mathcal{Q}_{2}} \sum_{a, b, x, y} B_{a b x y} P_{c^{\prime} \mid z^{\prime}}(a b \mid x y) \max P\left(c^{\prime} \mid z^{\prime}\right) \\
& =\max _{\vec{P}_{c^{\prime} \mid z^{\prime}} \in \mathcal{Q}_{2}} \sum_{a, b, x, y} B_{a b x y} P_{c^{\prime} \mid z^{\prime}}(a b \mid x y)=\beta_{\mathcal{Q}},
\end{aligned}
$$

where the first inequality follows from the fact that an independent maximization over $\vec{P}_{c^{\prime} \mid z^{\prime}} \in \mathcal{Q}_{2}$ and $P\left(c^{\prime} \mid z^{\prime}\right)$ is, in principle, less constraining than a maximization over all tripartite quantum distributions $\left\{P\left(a b c^{\prime} \mid x y z^{\prime}\right)\right\}$, the second-last equality follows from the fact that $P\left(c^{\prime} \mid z^{\prime}\right) \leqslant 1$ for legitimate marginal probability distributions, and the last equality follows from the fact that any bipartite quantum correlation can be seen as the marginalization of a tripartite one.

To complete the proof, note that the inequality $\beta_{\mathcal{Q}}^{\mathrm{LP}} \leqslant \beta_{\mathcal{Q}}$ can indeed be saturated if the three parties share a state of the form $\left|\psi_{123}\right\rangle=\left|\psi_{12}^{*}\right\rangle \otimes\left|\psi_{3}\right\rangle$ (with $\left|\psi_{3}\right\rangle$ being some arbitrary pure state that is in the possession of the third party) while employing the local measurements:

$$
M_{a \mid x}^{(1)}=M_{a \mid x}^{(1 *)}, \quad M_{b \mid y}^{(2)}=M_{b \mid y}^{(2 *)}, \quad M_{c \mid z^{\prime}}^{(3)}=\mathbf{1} \delta_{c, c^{\prime}},
$$

where $\left|\psi_{12}^{*}\right\rangle,\left\{M_{a \mid x}^{(1 *)}\right\}_{a, x},\left\{M_{b \mid y}^{(2 *)}\right\}_{b, y}$ constitute a maximizer for the (original) Bell inequality of Eq. (16), i.e.,

$$
\begin{aligned}
\beta_{\mathcal{Q}} & =\max _{\vec{P} \in \mathcal{Q}_{2}} \sum_{a, b, x, y} B_{a b x y} P(a b \mid x y) \\
& =\sum_{a, b, x, y} B_{a b x y} P^{*}(a b \mid x y) \\
& =\sum_{a, b, x, y} B_{a b x y}\left\langle\psi_{12}^{*}\left|M_{a \mid x}^{(1 *)} \otimes M_{b \mid y}^{(2 *)}\right| \psi_{12}^{*}\right\rangle .
\end{aligned}
$$

\section{Implications on self-testing}

As an implication of the above observation, we obtain the following result in the context of quantum theory.

Corollary 2. If a Bell inequality self-tests $|\tilde{\psi}\rangle$ and some reference POVMs $\left\{\tilde{M}_{a \mid x}^{(1)}\right\}_{a, x},\left\{\tilde{M}_{b \mid y}^{(2)}\right\}_{b, y}$ etc., then the maximal quantum violation of any Bell inequality obtained therefrom via party lifting also self-tests the same state and the same local POVMs for an appropriate subset of parties.

Proof. In the following, we use inequality (17) to illustrate how the proof works in the tripartite case. Note from Eq. (20) that when the party-lifted Bell inequality of Eq. (17) is violated to its quantum maximum $\beta_{\mathcal{Q}}$, the marginal distribution of Charlie necessarily satisfies $P\left(c^{\prime} \mid z^{\prime}\right)=1$. It then follows from Eq. (19) that

$$
P\left(a b c^{\prime} \mid x y z^{\prime}\right)=P_{c^{\prime} \mid z^{\prime}}(a b \mid x y) P\left(c^{\prime} \mid z^{\prime}\right), \quad \forall a, b, x, y .
$$

Furthermore, from Eq. (20), this tripartite distribution gives the quantum bound of inequality (17) only if the marginal distributions $P_{c^{\prime} \mid z^{\prime}}(a b \mid x y)=P(a b \mid x y)$ of Eq. (23) violate the original Bell inequality of Eq. (16) to its quantum bound. Therefore, if the original Bell inequality self-tests the twopartite entangled state $\left|\tilde{\psi}_{12}\right\rangle$, then for any tripartite density matrix $\rho_{123}$ leading to the quantum maximum of inequality Eq. (17), there must exist a local isometry $\Phi=\Phi_{1} \otimes \Phi_{2}$ 
such that

$$
\begin{aligned}
& \Phi \operatorname{tr}_{3}\left(\rho_{123}\right) \Phi^{\dagger}=|\tilde{\psi}\rangle\langle\tilde{\psi}| \otimes \rho_{\text {aux }}, \\
& \Phi\left[M_{a \mid x}^{(1)} \otimes M_{b \mid y}^{(2)} \operatorname{tr}_{3}\left(\rho_{123}\right)\right] \Phi^{\dagger} \\
& =\left(\tilde{M}_{a \mid x}^{(1)} \otimes \tilde{M}_{b \mid y}^{(2)}|\tilde{\psi}\rangle\langle\tilde{\psi}|\right) \otimes \rho_{\text {aux }},
\end{aligned}
$$

where $\rho_{\text {aux }}$ is some auxiliary density matrix acting on other degrees of freedom of Alice and Bob's subsystem. In other words, if the quantum maximum of the original Bell inequality can be used to self-test $|\tilde{\psi}\rangle$ and reference POVMs $\left\{\tilde{M}_{a \mid x}^{(1)}\right\}_{a, x},\left\{\tilde{M}_{b \mid y}^{(2)}\right\}_{b, y}$, so does the quantum maximum of the party-lifted Bell inequality.

\section{Implications on device-independent certification of entanglement depth}

In Theorem 2 of [59], it was shown that if

$$
\sum_{k_{1} \ldots k_{n}, j_{1} \ldots j_{n}} B_{\vec{k}, \vec{j}} P(\vec{k} \mid \vec{j}) \stackrel{\mathcal{R}_{n, \ell}}{\leqslant} 0
$$

is satisfied by an $n$-partite resource $\mathcal{R}$ (quantum or nonsignaling) that has a group size of $\ell$, its lifting to $(n+h)$ parties also holds for the same kind of resource of group size $\ell$ :

$$
\sum_{k_{1} \cdots k_{n}, j_{1} \cdots j_{n}} B_{\vec{k}, \vec{j}} P(\vec{k}, \vec{o} \mid \vec{j}, \vec{s}) \stackrel{\mathcal{R}_{n+h, \ell}}{\leqslant} 0,
$$

where $\vec{o}(\vec{s})$ is any fixed, but arbitrary string of outputs (inputs) for the $h$ additional parties. For the case of $\ell=n$, the above result reduces to observation 1 discussed in Sec. III C 1.

When the considered resource is restricted to shared quantum correlations and if $\ell<n$, inequalities (25) and (26) are instances of so-called device-independent witnesses for entanglement depth [26], i.e., Bell-like inequalities capable of certifying - directly from the observed correlation-a lower bound on the entanglement depth [60] of the measured system. More specifically, if the observed quantum value of the left-hand side of Eqs. (25) or (26) is greater than 0, then one can certify that the locally measured quantum state must have an entanglement depth of at least $\ell+1$.

Although the above result of [59] can be applied to an arbitrary number of $(n+h)$ parties, observation 1 implies that if the seed inequality is that applicable to an $n$-partite Bell scenario, the extended scenario can never be used to certify an entanglement depth larger than $n$. This follows from the fact that the maximal quantum value of these party-lifted Bell-like inequalities is the same as the original Bell-like inequality [cf. Eq. (25)], and is already attainable using a quantum state of entanglement depth $n$.

\section{CONCLUDING REMARKS}

Lifting, as introduced by Pironio [41], is a procedure that allows one to systematically construct Bell inequalities for all Bell scenarios starting from a Bell inequality applicable to a simpler scenario. It is known that Pironio's lifting preserves the facet-defining property of Bell inequalities, and thus lifted Bell inequalities (in particular, those lifted from the $\mathrm{CHSH}$ Bell inequality) can be found in all nontrivial Bell scenarios. In this work, we show that lifting leaves both the maximal quantum value and the maximal nonsignaling value of Bell inequalities unchanged.

Naturally, one may ask whether the quantum state and local measurements maximally violating a lifted Bell inequality are related to that of the original Bell inequality. Indeed, we show that Pironio's lifting also preserves the self-testability of a quantum state. Hence, the quantum state maximally violating a lifted Bell inequality is-modulo irrelevant local degrees of freedom - the same as that of the original inequality. Likewise, the self-testability of given local measurements is preserved using any but the outcome-lifting procedure.

The maximizers of lifted Bell inequalities are, as we show, generally not unique. Consequently, it is impossible to use the observed quantum value of such an inequality to self-test both the underlying state and all the local measurements: in the case of an input-lifted Bell inequality, no conclusions can be drawn regarding the additional measurements that do not appear in the inequality; in the case of a party-lifted Bell inequality, nothing can be said about the measurements of the additional party; in the case of an output-lifted Bell inequality, the self-testing of all the local POVM elements is impossible, but the self-testing of their combined effect seems possible. In fact, our numerical results (see Appendix C) suggest that such a self-testing is just as robust as the original Bell inequality. Thus, Bell inequalities lifted from $\mathrm{CHSH}$ serve as generic examples whose maximal quantum violation can be used to self-test a state, but not the underlying measurements in its entirety.

Notice also that the nonuniqueness mentioned above evidently becomes more and more pronounced as the number of "irrelevant" degrees of freedom increases, for example, by repeatedly applying lifting to a given Bell inequality. Since only correlation $\vec{P}$ belonging to the boundary of $\mathcal{Q}$ could violate a linear Bell inequality maximally, as the complexity of the Bell scenario (say, in terms of the number of measurements, outcomes, or parties) increases, it is conceivable that one can always find a flat boundary of $\mathcal{Q}$ (corresponding to those of the lifted Bell inequality) with increasing dimension. Proving this statement rigorously and finding the exact scaling of the dimension of these flat boundaries would be an interesting direction to pursue for future research.

Besides, it will be interesting to see-in comparison with the original Bell inequality - whether the robustness in selftesting that we have observed for a particular version of the outcome-lifted CHSH Bell inequality is generic. From our example for the outcome-lifted CHSH inequality, it becomes clear that self-testing of the combined POVM elements is (sometimes) possible even if the self-testing of all individual POVM elements is not. This possibility opens another direction of research in the context of self-testing. In addition, our results also prompted the following question: does there exist a physical situation (say, the observation of the maximal quantum value of some Bell inequality) where the underlying measurements can be self-tested, but not the underlying state? Since the self-testing of measurements is seemingly more demanding than that for a quantum state, it is conceivable that no such examples can be constructed. Proving that this is indeed the case, however, clearly lies beyond the scope of the present paper. 
Note added. While completing this manuscript, we became aware of the work of [61] which also discusses, among others, extensively the properties of liftings, as well as the work of [62], which exhibits examples of quantum correlations that can only be used to self-test the measured quantum state but not the underlying measurements.

\section{ACKNOWLEDGMENTS}

We thank J.-D. Bancal, J. Kaniewski, D. Rosset, and Ivan Šupić for useful discussions. This work was supported by the Foundation for the Advancement of Outstanding Scholarship, Taiwan as well as the Ministry of Science and Technology, Taiwan (Grants No. 104-2112-M-006-021-MY3, No. 1072112-M-006-005-MY2, No. 107-2627-E-006-001, No. 1082811-M-006-501, and No. 108-2811-M-006-516).

\section{APPENDIX A: EXAMPLES OF $\overrightarrow{\boldsymbol{P}} \in \mathcal{Q}$ VIOLATING LIFTED BELL INEQUALITIES MAXIMALLY}

To illustrate the nonunique nature of the maximizers of lifted Bell inequalities, consider the CHSH Bell inequality [3],

$$
\sum_{x, y, a, b=0,1}(-1)^{x y+a+b} P(a b \mid x y) \stackrel{\mathcal{L}}{\leqslant} 2,
$$

as our seed inequality. Since this is a Bell inequality defined in the simplest Bell scenario (with two binary output per party), its liftings can be found in all nontrivial Bell scenarios.

The quantum bound and nonsignaling bound of the above Bell inequality are given, respectively, by $\beta_{\mathcal{Q}}=2 \sqrt{2}$ and $\beta_{\mathcal{N}}=4$. It is known [9] that the maximal quantum violation of the CHSH inequality can be used to self-test (up to local isometry) the two-qubit maximally entangled state $\left|\phi^{+}\right\rangle=$ $(|00\rangle+|11\rangle) / \sqrt{2}$ and the Pauli observables $\left\{\sigma_{z}, \sigma_{x}\right\}$ on one side and the Pauli observables $\left\{\left(\sigma_{x}+\sigma_{z}\right) / \sqrt{2},\left(\sigma_{x}-\sigma_{z}\right) / \sqrt{2}\right\}$ on the other. Thus, the correlation that gives the quantum maximum of the inequality (A1) is unique and is given by

$$
P_{Q}(a b \mid x y)=\frac{1}{4}+(-1)^{a+b+x y} \frac{\sqrt{2}}{8}, \quad a, b, x, y \in\{0,1\},
$$

where the +1 outcome of the observables is identified with the zeroth outcome in the conditional outcome probability distributions.

\section{Lifting of inputs}

For input lifting, consider now a bipartite Bell scenario where Bob has instead three binary inputs $(y=0,1,2)$. In this new Bell scenario, the following Bell inequality:

$$
I_{2}^{\mathrm{LI}-\mathrm{CHSH}}:=\sum_{x, y, a, b=0,1}(-1)^{x y+a+b} \tilde{P}(a b \mid x y) \stackrel{\mathcal{L}}{\leqslant} 2,
$$

with $\{\tilde{P}(a b \mid x y)\}_{a, b, x=0,1, y=0,1,2}$, can be obtained by applying input lifting to inequality (A1).

To illustrate the nonuniqueness of its maximizers, one may employ, e.g., either of the two trivial measurements for the third measurement $(y=2)$ :

$$
M_{b \mid 2}^{(2)}=\mathbf{1} \delta_{b, 0} \quad \text { or } \quad M_{b \mid 2}^{(2)}=\mathbf{1} \delta_{b, 1} .
$$

Correspondingly, one obtains, in addition to [cf. Eq. (A2)]

$$
\tilde{P}_{Q_{1}}^{\mathrm{LI}}(a b \mid x, y)=\tilde{P}_{Q_{2}}^{\mathrm{LI}}(a b \mid x, y)=P_{Q}(a, b \mid x, y)
$$

for $a, b, x, y=0,1$, the distributions

$$
\tilde{P}_{Q_{1}}^{\mathrm{LI}}(a b \mid x y)=\frac{1}{2} \delta_{b, 0} \quad \text { and } \quad \tilde{P}_{Q_{2}}^{\mathrm{LI}}(a b \mid x, y)=\frac{1}{2} \delta_{b, 1},
$$

for $a, b, x=0,1$ but $y=2 .^{2}$

It is then easily verified that both these correlations violate inequality (A3) to its quantum maximum of $2 \sqrt{2}$. In fact, since the Bell expression of Eq. (A3) is linear in $\tilde{P}$, it follows that an arbitrary convex combination of $\tilde{P}_{Q_{1}}^{\mathrm{LI}}$ and $\tilde{P}_{Q_{2}}^{\mathrm{LI}}$,

$$
\tilde{P}(a b \mid x y)=p \tilde{P}_{Q_{1}}^{\mathrm{LI}}(a b \mid x y)+(1-p) \tilde{P}_{Q_{2}}^{\mathrm{LI}}(a b \mid x y),
$$

where $0 \leqslant p \leqslant 1$, must also give the maximal quantum value of Bell inequality (A3). Moreover, from the convexity of the set of quantum correlations $\mathcal{Q}$, we know that an arbitrary convex combination of the two correlations given above is also quantum realizable. Geometrically, this means that the set of $\tilde{P} \in \mathcal{Q}$ defined by Eq. (A6) forms a one-dimensional flat region of the quantum boundary. In Fig. 4, we show a two-dimensional slice on the space of correlations spanned by $\tilde{P}_{Q_{1}}^{\mathrm{LO}}, \tilde{P}_{Q_{2}}^{\mathrm{LO}}$, and the uniform distribution $\tilde{P}_{0}$. Note that on this peculiar slice, even $\mathcal{Q}$ appears to be a polytope.

\section{Lifting of outcomes}

For output lifting, consider a bipartite Bell scenario where Bob's measurements have instead three outcomes $(b=$ $0,1,2)$. In this Bell scenario, the following Bell inequality:

$$
I_{2}^{\mathrm{LO}-\mathrm{CHSH}}:=\sum_{x, y, a=0,1} \sum_{b=0,1,2}(-1)^{x y+a+b} P(a b \mid x y) \stackrel{\mathcal{L}}{\leqslant} 2,
$$

can be obtained by applying outcome lifting to the $b=0$ outcome of Bob's measurements in inequality (A1). Here, $b=2$ is the new outcome.

Indeed, it is readily seen that the two correlations,

$$
\begin{aligned}
& P_{Q_{1}}^{\mathrm{LO}}(a b \mid x y)=\left[\frac{1}{4}+(-1)^{a+b+x y} \frac{\sqrt{2}}{8}\right]\left(1-\delta_{b, 2}\right), \\
& P_{Q_{2}}^{\mathrm{LO}}(a b \mid x y)=\left[\frac{1}{4}+(-1)^{a+b+x y} \frac{\sqrt{2}}{8}\right]\left(1-\delta_{b, 0}\right),
\end{aligned}
$$

as well as

$$
\begin{aligned}
& P_{Q_{3}}^{\mathrm{LO}}(a b \mid x y)=P_{Q_{1}}^{\mathrm{LO}}(a b \mid x y) \delta_{y, 0}+P_{Q_{2}}^{\mathrm{LO}}(a b \mid x y) \delta_{y, 1}, \\
& P_{Q_{4}}^{\mathrm{LO}}(a b \mid x y)=P_{Q_{1}}^{\mathrm{LO}}(a b \mid x y) \delta_{y, 1}+P_{Q_{2}}^{\mathrm{LO}}(a b \mid x y) \delta_{y, 0},
\end{aligned}
$$

all give rise to the quantum maximum of $2 \sqrt{2}$ for the Bell inequality of Eq. (A7).

To see that they are indeed quantum realizable, we note that the correlation given in Eq. (A8) can be produced by employing the quantum strategy used to produce the correlation given in Eq. (A2). By construction, Bob's measurement only produces two outcomes labeled by $b=0$ and $b=1$, and thus

\footnotetext{
${ }^{2}$ More abstractly, these correlations can also be obtained from Eq. (A2) by applying input operations as given by Eq. (10) in [55].
} 
the $b=2$ outcome never appears, as required in Eq. (A8). To obtain the correlation given in Eq. (A9), one may start from the correlation of Eq. (A8) and apply the classical relabeling of $b=0 \leftrightarrow b=2$.

Similarly, the two correlations of Eq. (A10) can be realized by first implementing the quantum strategy that realizes $\vec{P}_{Q_{1}}^{\mathrm{LO}}$, followed by applying the classical relabeling of $b=0 \leftrightarrow b=$ 2 depending on whether $y=0$ or $y=1 .{ }^{3}$ Since $\left\{\vec{P}_{Q_{i}}^{\mathrm{LO}}\right\}_{i=1}^{4}$ forms a linearly independent set, and an arbitrary convex combination of them also gives the quantum bound, we thus see that the quantum face ${ }^{4}$ of the outcome-lifted inequality of (A7) is (at least) three dimensional.

\section{Lifting of party}

Geometrically, party lifting also introduces degeneracy in the maximizers of a Bell inequality. For example, a possible party lifting of the CHSH Bell inequality of Eq. (A1) to the three-party, two-input, two-output Bell scenario reads as

$$
\sum_{x, y, a, b=0,1}\left\{\left[(-1)^{x y+a+b}-\frac{1}{2}\right] P(a b 0 \mid x y 0)\right\} \stackrel{\mathcal{L}}{\leqslant} 0 .
$$

As mentioned in the proof of Corollary 2, maximizers of this Bell inequality must be such that the bipartite marginal distribution $P(a b \mid x y)$ violates CHSH Bell inequality maximally while the marginal distribution for the third party must satisfy $P(0 \mid 0)=1$. However, these conditions do not impose any constraint on the other marginal distribution $P(c \mid z)$ for $z \neq 0$. In particular, both the choice of $P(c \mid 1)=\delta_{c, 0}$ and $P(c \mid 1)=\delta_{c, 1}$ would fulfill the above requirement. As such, although the quantum face of the CHSH Bell inequality is a point in the correlation space, the quantum face of the party-lifted Bell inequality of Eq. (A11) has become one dimensional.

\section{APPENDIX B: QUANTUM REALIZABILITY OF DISTRIBUTIONS OBTAINED BY GROUPING AND SPLITTING OUTCOMES}

In this Appendix, we provide the details showing how one can-while preserving between the quantum violation of a Bell inequality and its outcome-lifted version-realize quantum mechanically a fewer-outcome (more-outcome) correlation if the original more-outcome (fewer-outcome) correlation is quantum.

\section{Grouping of outcomes}

Suppose that the joint probabilities $P\left(a b^{\prime} \mid x y^{\prime}\right)$ and $P\left(a u \mid x y^{\prime}\right)$ on the right-hand side of Eq. (10) are realized by a quantum state $\rho_{12}$ with the POVM $\left\{M_{a \mid x}^{(1)}\right\}_{a, x}$ on Alice's side and $\left\{M_{b \mid y}^{(2)}\right\}_{b, y}$ on Bob's side; cf. Eq. (4). Then the joint probabilities $\tilde{P}\left(a b^{\prime} \mid x y^{\prime}\right)$ appearing on the left-hand-side of Eq. (10), which corresponds to an effective $v$-outcome distribution, is

\footnotetext{
${ }^{3}$ This belongs to the class of outcome operation given by Eq. (9) in [55].

${ }^{4}$ The set of quantum correlations that gives the quantum bound of a Bell inequality is called the quantum face; see [44].
}

realizable by the same quantum state $\rho_{12}$ with the same POVM $\left\{M_{a \mid x}^{(1)}\right\}_{a, x}$ on Alice's side and the following POVM $\tilde{M}_{b \mid y}^{(2)}$ on Bob's side: 5

$$
\begin{aligned}
& \tilde{M}_{b \mid y}^{(2)}=M_{b \mid y}^{(2)} \quad y \neq y^{\prime}, \\
& \tilde{M}_{b \mid y^{\prime}}^{(2)}=M_{b \mid y^{\prime}}^{(2)} \quad b \notin\left\{b^{\prime}, u\right\}, \\
& \tilde{M}_{b^{\prime} \mid y^{\prime}}^{(2)}=M_{b^{\prime} \mid y^{\prime}}^{(2)}+M_{u \mid y^{\prime}}^{(2)} .
\end{aligned}
$$

With this choice, it follows from

$$
\tilde{P}\left(a b^{\prime} \mid x y^{\prime}\right)=\operatorname{tr}\left(M_{a \mid x}^{(1)} \otimes \tilde{M}_{b^{\prime} \mid y^{\prime}}^{(2)} \rho_{12}\right),
$$

Eq. (B1), and

$$
\begin{aligned}
\operatorname{tr}\left(M_{a \mid x}^{(1)} \otimes \tilde{M}_{b^{\prime} \mid y^{\prime}}^{(2)} \rho_{12}\right)= & \operatorname{tr}\left(M_{a \mid x}^{(1)} \otimes M_{b^{\prime} \mid y^{\prime}}^{(2)} \rho_{12}\right) \\
& +\operatorname{tr}\left(M_{a \mid x}^{(1)} \otimes M_{u \mid y^{\prime}}^{(2)} \rho_{12}\right)
\end{aligned}
$$

that Eq. (10) is satisfied, and hence that the violationpreserving fewer-outcome correlation is indeed attainable by coarse graining, i.e., the grouping of outcomes.

\section{Splitting of outcomes}

On the other hand, if we instead start from the original (fewer-outcome) Bell scenario, then the joint probabilities appearing on the right-hand side of Eq. (12), which corresponds to a $v+1$-outcome distribution, can be realized by, e.g., employing the same quantum state $\rho_{12}$ with the same POVM $\left\{M_{a \mid x}^{(1)}\right\}_{a, x}$ on Alice's side and the following POVM $\tilde{M}_{b \mid y}^{(2)}$ on Bob's side:

$$
\begin{aligned}
M_{b \mid y}^{(2)} & =\tilde{M}_{b \mid y}^{(2)} \quad y \neq y^{\prime}, \\
M_{b \mid y^{\prime}}^{(2)} & =\tilde{M}_{b \mid y^{\prime}}^{(2)} \quad b \notin\left\{b^{\prime}, u\right\}, \\
M_{b^{\prime} \mid y^{\prime}}^{(2)} & =p \tilde{M}_{b^{\prime} \mid y^{\prime}}^{(2)}, \quad M_{u \mid y^{\prime}}^{(2)}=(1-p) \tilde{M}_{b^{\prime} \mid y^{\prime}}^{(2)},
\end{aligned}
$$

for arbitrary $0 \leqslant p \leqslant 1$. The positivity of the left-hand side of Eq. (B4) and their normalization are evident from their definition. Moreover, using Eq. (B3) and

$$
\widehat{P}(a b \mid x y)=\operatorname{tr}\left(M_{a \mid x}^{(1)} \otimes M_{b^{\prime} \mid y^{\prime}}^{(2)} \rho_{12}\right),
$$

it is easy to see that Eq. (12) holds with the assignment given in Eq. (B4). Hence, the POVM given by the left-hand side of Eq. (B4) indeed realizes the required violation-preserving more-outcome correlation by splitting the $b^{\prime}$ outcome of Bob's $y^{\prime}$ th measurement.

\section{APPENDIX C: ROBUST SELF-TESTING BASED ON THE QUANTUM VIOLATION OF THE OUTCOME-LIFTED CHSH INEQUALITY}

We show in this Appendix that self-testing via the quantum violation of the outcome-lifted inequality of Eq. (A7) is robust. In this regard, note that the maximal quantum violation of inequality (A7) can also be achieved by Alice and Bob

\footnotetext{
${ }^{5}$ That $\tilde{M}_{b \mid y}^{(2)}$ satisfies both the positivity constraints and the normalization constraints is evident from Eq. (B1).
} 
sharing the following two-qubit maximally entangled state:

$$
|\tilde{\psi}\rangle=\cos \frac{\pi}{8} \frac{|00\rangle-|11\rangle}{\sqrt{2}}+\sin \frac{\pi}{8} \frac{|01\rangle+|10\rangle}{\sqrt{2}},
$$

while performing the optimal qubit measurements for Alice:

$$
\begin{array}{ll}
\tilde{M}_{0 \mid 0}^{(1)}=\frac{1}{2}\left(\mathbb{1}+\sigma_{z}\right), & \tilde{M}_{1 \mid 0}^{(1)}=\frac{1}{2}\left(\mathbb{1}-\sigma_{z}\right), \\
\tilde{M}_{0 \mid 1}^{(1)}=\frac{1}{2}\left(\mathbb{1}+\sigma_{x}\right), & \tilde{M}_{1 \mid 1}^{(1)}=\frac{1}{2}\left(\mathbb{1}-\sigma_{x}\right),
\end{array}
$$

and for Bob:

$$
\begin{array}{lll}
\tilde{M}_{0 \mid 0}^{(2)}=E_{0 \mid 0}, & \tilde{M}_{1 \mid 0}^{(2)}=\frac{1}{2}\left(\mathbb{1}-\sigma_{z}\right), & \tilde{M}_{2 \mid 0}^{(2)}=E_{2 \mid 0}, \\
\tilde{M}_{0 \mid 1}^{(2)}=E_{0 \mid 1}, & \tilde{M}_{1 \mid 1}^{(2)}=\frac{1}{2}\left(\mathbb{1}-\sigma_{x}\right), & \tilde{M}_{2 \mid 1}^{(2)}=E_{2 \mid 1},
\end{array}
$$

where $\left\{E_{b \mid y}\right\}_{b=0,2}$ are any valid POVM elements satisfying $\sum_{b=0,2} E_{b \mid y}=\mathbb{1}-M_{1 \mid y}^{(2)}$ for all $y$.

Notice that Eqs. (5) and (6) only hold for the case of perfect self-testing of the reference state and reference measurements. To demonstrate robust self-testing for the above reference state $|\tilde{\psi}\rangle$ and reference measurements $\left\{\tilde{M}_{a \mid x}^{(1)}\right\}_{a, x},\left\{\tilde{M}_{b \mid y}^{(2)}\right\}_{b, y}$, we follow the approach of [56] to arrive at statements saying that if the observed quantum violation of inequality (A7) is close to its maximal value, then (1) the measured system contains some degrees of freedom that have a high fidelity with respect to the reference state $|\tilde{\psi}\rangle$, and (2) with high probability, the uncharacterized measurement devices function like $\left\{\tilde{M}_{a \mid x}^{(1)}\right\}_{a, x}$, $\left\{\tilde{M}_{b \mid y}^{(2)}\right\}_{b, y}$ acting on the same degrees of freedom.

\section{Robust self-testing of the reference state}

To this end, we shall make use of the swap method proposed in [56]. The key idea is to introduce local swap operators $\Phi_{1}, \Phi_{2}$ so that the state acting on Alice's and Bob's Hilbert space (of unknown dimension) gets swapped locally with some auxiliary states of trusted Hilbert space dimension (qubit in our case). To better understand how this works, let us first consider an example with characterized devices before proceeding to the case where the devices are uncharacterized.

For this purpose, let us concatenate the following controlled-not (CNOT) gates,

$$
\begin{aligned}
& U_{1}=\mathbb{1} \otimes|0\rangle\left\langle 0\left|+\sigma_{x} \otimes\right| 1\right\rangle\langle 1|, \\
& V_{1}=|0\rangle\langle 0|\otimes \mathbb{1}+| 1\rangle\langle 1| \otimes \sigma_{x},
\end{aligned}
$$

to obtain the (two-qubit) swap gate $\Phi_{1}=U_{1} V_{1} U_{1}$ acting on $\mathcal{H}_{A} \otimes \mathcal{H}_{A^{\prime}}$ (see Sec. II B). We may define a swap operator $\Phi_{2}=U_{2} V_{2} U_{2}$ acting on Bob's systems in exactly the same way. Importantly, one notices from Eqs. (C2) and (C3) that it is possible to express the individual unitaries in terms of the POVM elements leading to the maximal quantum violation of inequality (A7). For example, one may take

$$
\sigma_{z}=\mathbb{1}-2 \tilde{M}_{1 \mid 0}^{(i)}, \quad \sigma_{x}=\mathbb{1}-2 \tilde{M}_{1 \mid 1}^{(i)} \quad \forall i=1,2 .
$$

Moreover, if we define the global swap gate by $\Phi=\Phi_{1} \otimes$ $\Phi_{2}$ and denote the state acting on $\mathcal{H}_{A} \otimes \mathcal{H}_{B}$ by $\rho_{12}$, then the "swapped" state is

$$
\rho^{\mathrm{SWAP}}:=\operatorname{tr}_{12}\left[\Phi\left(|0\rangle\left\langle 0\left|\otimes \rho_{12} \otimes\right| 0\right\rangle\langle 0|\right) \Phi^{\dagger}\right],
$$

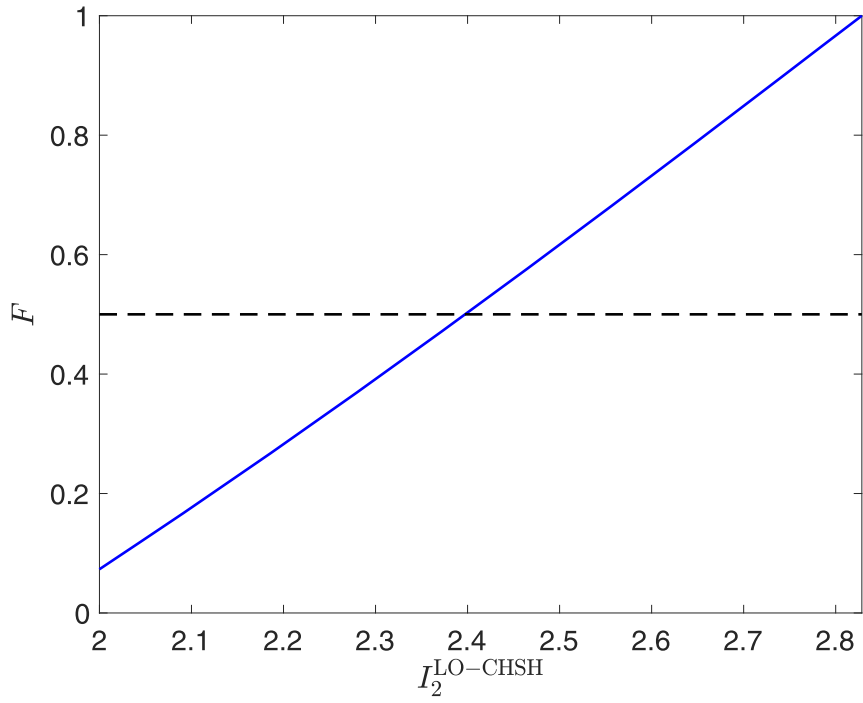

FIG. 5. Lower bounds on the fidelity as a function of the value of the outcome-lifted CHSH inequality $I_{2}^{\mathrm{LO}-\mathrm{CHSH}}$. The results are obtained by solving the semidefinite program described in Eq. (C8).

where $\operatorname{tr}_{12}$ represents a partial trace over the Hilbert space of $\mathcal{H}_{A} \otimes \mathcal{H}_{B}$. When $\Phi$ is exactly the swap gate defined above via Eq. (C4), $\rho^{\text {SWAP }}$ is exactly $\rho_{12}$. Thus, the fidelity between $\rho^{\mathrm{SWAP}}$ and the reference state $|\tilde{\psi}\rangle: F=\left\langle\tilde{\psi}\left|\rho^{\mathrm{SWAP}}\right| \tilde{\psi}\right\rangle$ provides a figure of merit on the similarity between (some relevant parts of) the shared state $\rho_{12}$ and the reference state $|\tilde{\psi}\rangle$.

To perform a device-independent characterization, the assumption of $\tilde{M}_{1 \mid x}^{(1)}$ and $\tilde{M}_{1 \mid y}^{(2)}$ is relaxed to unknown projectors $M_{1 \mid x}^{(1)}$ and $M_{1 \mid y}^{(2)}$ (acting on Hilbert space of arbitrary dimensions), and the corresponding "CNOT" gates become

$$
\begin{aligned}
& U_{i}=\mathbb{1} \otimes|0\rangle\left\langle 0\left|+\left(\mathbb{1}-2 M_{1 \mid 1}^{(i)}\right) \otimes\right| 1\right\rangle\langle 1|, \\
& V_{i}=\left(\mathbb{1}-M_{1 \mid 0}^{(i)}\right) \otimes \mathbb{1}+M_{1 \mid 0}^{(i)} \otimes \sigma_{x},
\end{aligned}
$$

for $i=1,2$. One can verify that the fidelity $F=\left\langle\tilde{\psi}\left|\rho^{\mathrm{SWAP}}\right| \tilde{\psi}\right\rangle$ is then a linear function of the moments such as $\left\langle M_{a \mid x}^{(1)} \otimes\right.$ $\left.M_{b \mid y}^{(2)}\right\rangle,\left\langle M_{a \mid x}^{(1)} \otimes M_{b \mid y}^{(2)} M_{b^{\prime} \mid y^{\prime}}^{(2)}\right\rangle$, etc., where $\langle\cdot\rangle:=\operatorname{tr}\left(\cdot \rho_{12}\right)$.

Thus, a lower bound on $F$ for any observed value of Bell inequality violation (without assuming the shared state or the measurements performed) can be obtained by solving the following semidefinite program:

$$
\begin{aligned}
\min & F, \\
\text { such that } & \Gamma^{S} \succeq 0, \\
& I_{2}^{\mathrm{LO}-\mathrm{CHSH}}=I_{2 \text {,obs }}^{\mathrm{LO}-\mathrm{CHSH}},
\end{aligned}
$$

where $\Gamma^{S}$ is any Navascués-Pironio-Acín-type [63] moment matrix that contains all the moments appearing in $F$. In our computation, we employed a moment matrix that is built from a sequence of operators $S$ that contain all operators from level $1+A B$ (or equivalently, level 1 from the hierarchy of Ref. [64]) and some additional operators from level 3.

Our results (see Fig. 5) clearly show that the self-testing property of $I_{2}^{\mathrm{LO}-\mathrm{CHSH}}$ with respect to the reference maximally entangled state $|\tilde{\psi}\rangle$ of Eq. (C1) is indeed robust. In other 
words, as long as the observed violation of $I_{2}^{\mathrm{LO}-\mathrm{CHSH}}$ is greater than $\approx 2.4$, one can still obtain a nontrivial lower bound on the fidelity $(>1 / 2)$ with respect to $|\tilde{\psi}\rangle$. Moreover, a separate computation using the original CHSH Bell inequality of Eq. (A1) (and the same level of approximation of $\mathcal{Q}$ ) gives-within the numerical precision of the solver-the same curve, thereby suggesting that the outcome-lifted Bell inequality of (A7) offers the same level of robustness as compared with its seed inequality.

\section{Robust self-testing of Alice's POVM}

Even though it is impossible to completely self-test all local measurements, robust self-testing of Alice's POVMas one would intuitively expect-can still be achieved. In particular, when the observed violation of $I_{2}^{\mathrm{LO}-\mathrm{CHSH}}$ is close to the quantum bound of $2 \sqrt{2}$, it must be the case that Alice's measurements (on the relevant degrees of freedom) indeed behave like measurements in the $\sigma_{z}$ and $\sigma_{x}$ basis, respectively, for $x=0,1$.

To that end, we again make use the swap method proposed in Ref. [56]. The idea is that if these measurements behave as expected, then their measurements on the auxiliary states swapped into the uncharacterized device, i.e., $\Phi_{1}(|\varphi\rangle)$-with $|\varphi\rangle$ being eigenstates of $\sigma_{z}$ and $\sigma_{x}$-should produce outcomes $a$ with statistics $\{P(a|x,| \varphi\rangle)\}$ satisfying

$P(0|0| 0\rangle,)=P(1|0| 1\rangle,)=P(0|1|+\rangle,)=P(1|1|-\rangle,)=1$.

Using the same swap operator defined via Eq. (C7), we get

$$
P(a|x,| \varphi\rangle)=\operatorname{tr}\left\{M_{a \mid x}^{(1)}\left[\Phi_{1}\left(\rho_{12} \otimes|\varphi\rangle\langle\varphi|\right) \Phi_{1}^{\dagger}\right]\right\},
$$

where we have, for simplicity, omitted the identity operator acting on Bob's system. Notice that the left-hand side of Eq. (C10) is again some linear combination of moments. Likewise for the following figure of merit [56]:

$$
\begin{aligned}
\tau= & \left.\frac{1}{2}[P(0|0,| 0\rangle)+P(1|0,| 1\rangle\right) \\
& +P(0|1,|+\rangle)+P(1|1,|-\rangle)]-1,
\end{aligned}
$$

which takes value between -1 and +1 . The maximum of +1 , in particular, happens only when Alice's POVM $M_{a \mid x}^{(1)}$ corresponds to measurements in $\sigma_{z}$ and $\sigma_{x}$, respectively, for $x=0,1$. $\tau$ therefore quantifies the extent to which the measurement devices function like the reference measurements. Given a violation of $I_{2}^{\mathrm{LO}-\mathrm{CHSH}}$, a lower bound on $\tau$ can thus be obtained by solving the following semidefinite program:

$$
\begin{aligned}
\min & \tau, \\
\text { such that } & \Gamma^{S} \succeq 0, \\
& I_{2}^{\mathrm{LO}-\mathrm{CHSH}}=I_{2 \text {,obs }}^{\mathrm{LO}-\mathrm{CHSH}} .
\end{aligned}
$$

The resulting lower bounds on $\tau$ are shown in Fig. 6. We see that the value of 1 is obtained when the maximal quantum value of $I_{2}^{\mathrm{LO}-\mathrm{CHSH}}$ is observed, which means that the reference measurements of Eq. (C2) are correctly certified in this case. For nonmaximal values of $I_{2}^{\mathrm{LO}-\mathrm{CHSH}}$, we see that $\tau$ decreases accordingly. Importantly, as pointed out in Ref. [56], the procedure of sending the prepared eigenstates into the swap gate is a virtual process that allows us to interpret the figure

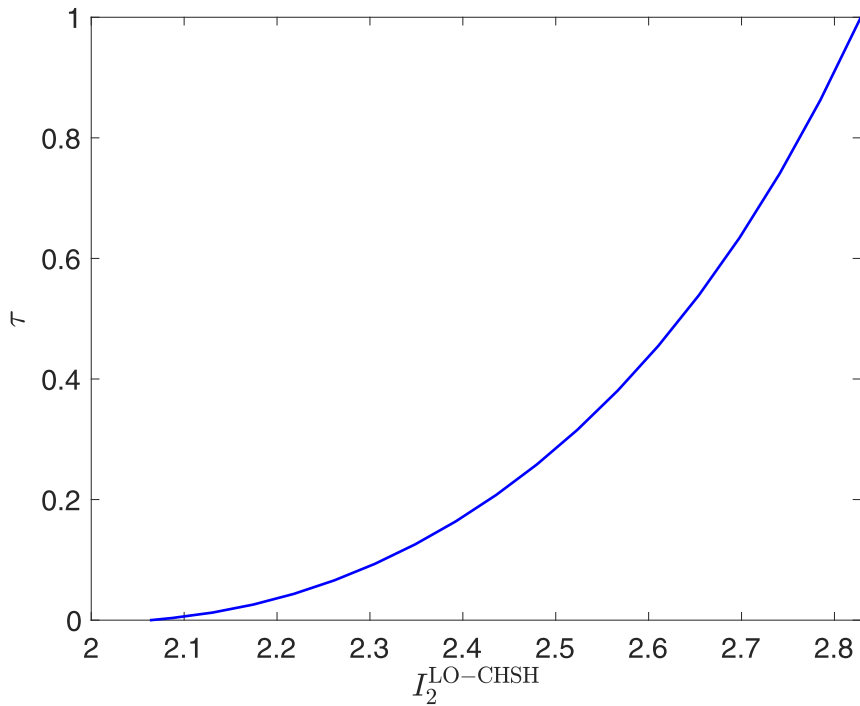

FIG. 6. Lower bounds on the figure of merit defined in Eq. (C11) as a function of the value of the outcome-lifted $\mathrm{CHSH}$ inequality $I_{2}^{\mathrm{LO}-\mathrm{CHSH}}$. The bounds are obtained by solving the semidefinite program described in Eq. (C12).

of merit operationally, but the result still holds without any assumption on the devices of interest.

\section{Partial but robust self-testing of Bob's POVMs}

Finally, we would like to show that the outcome-lifted CHSH inequality of Eq. (A7) can also be used for a "partial" self-testing of Bob's optimal measurements. The steps are the same as those described in the self-testing of Alice's

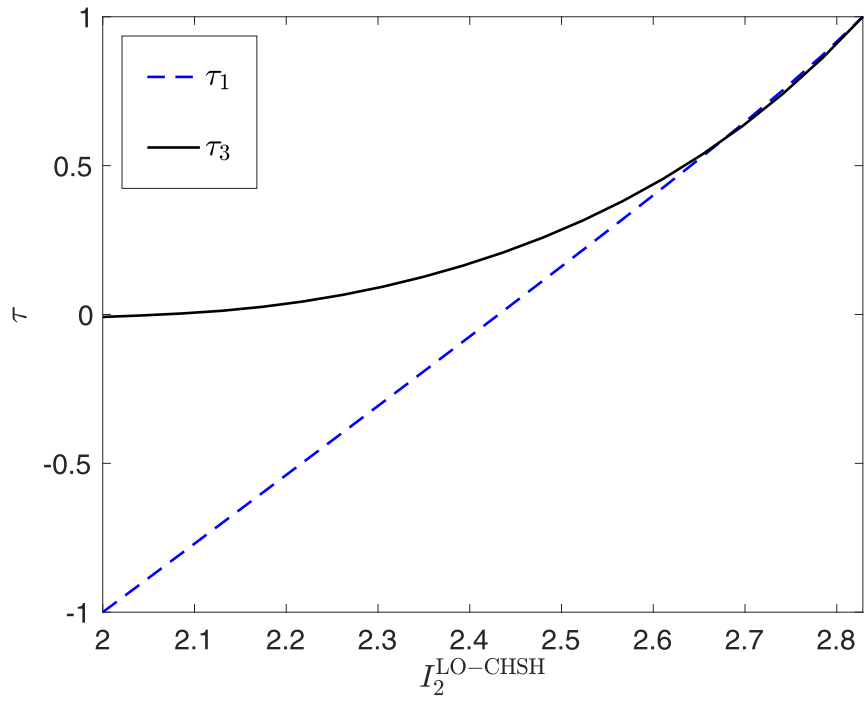

FIG. 7. Lower bounds on the figures of merit defined in Eqs. (C13) and (C14) as a function of the value of the outcome-lifted CHSH inequality $I_{2}^{\mathrm{LO}-\mathrm{CHSH}}$. The bounds are obtained by solving the semidefinite program described in Eq. (C12) with the appropriate figure of merit. The two figures of merit, as explained in the text, reflect different aspects of the self-testability of Bob's measurements. 
measurements. That is, the eigenstates of $\sigma_{z}$ and $\sigma_{x}$ are sent to the swap gate before Bob performs his measurements $\left\{M_{b \mid y}^{(2)}\right\}$.

To this end, we define the analog of Eq. (C11) as

$$
\begin{aligned}
\tau_{3}= & \left.\left.\frac{1}{2}[P(0|0,| 0\rangle)+P(1|0,| 1\rangle\right)+P(2|0,| 0\rangle\right) \\
& +P(0|1,|+\rangle)+P(1|1,|-\rangle)+P(2|1,|+\rangle)]-1,
\end{aligned}
$$

and introduce a further figure of merit

$$
\left.\left.\tau_{1}=P(1|0,| 0\rangle\right)+P(1|1,|+\rangle\right)-1
$$

to self-test only the POVM element corresponding to Bob's outcome 1 for both measurements.

Thus, $\tau_{3}$ takes into account of Bob's all measurements outcomes while $\tau_{1}$ only involves the second measurement outcome. All these figures of merit range from -1 to +1 , and +1 is recovered for (1) $\tau_{3}$ if Bob's measurement device acts on the swapped eigenstate according to Eq. (C3), (2) $\tau_{1}$ if Bob's measurement device acts on the swapped eigenstate in such a way that the second POVM element for each measurement functions according to Eq. (C3). In other words, the value of $\tau_{1}$ measures the extent to which $M_{1 \mid y}^{(2)}$ behaves according to that prescribed in Eq. (C3), while the value of $\tau_{3}$ further indicates if the combined effect of $M_{0 \mid y}^{(2)}+M_{2 \mid y}^{(2)}$ also behaves according to that prescribed in Eq. (C3).

By solving the semidefinite program of Eq. (C12) using the appropriate objective functions, we obtained lower bounds on each figure of merit as a function of the quantum violation of $I_{2}^{\mathrm{LO}-\mathrm{CHSH}}$. As shown in Fig. 7 , the bounds on $\tau_{3}$ and $\tau_{1}$ when $I_{2}^{\mathrm{LO}-\mathrm{CHSH}}$ take their maximal value successfully selftests, respectively, the combined effect of $M_{0 \mid y}^{(2)}+M_{2 \mid y}^{(2)}$ as well as that of $M_{1 \mid y}^{(2)}$. In summary, for the outcome-lifted CHSH inequality of Eq. (A7), where the first outcome is lifted in each of Bob's measurement, it is still possible to self-test Alice's optimal measurements and the overall behavior of Bob's measurements.
[1] A. Einstein, B. Podolsky, and N. Rosen, Can quantummechanical description of physical reality be considered complete? Phys. Rev. 47, 777 (1935).

[2] J. S. Bell, On the Einstein Podolsky Rosen paradox, Physics (Long Island City, N. Y.) 1, 195 (1964).

[3] J. F. Clauser, M. A. Horne, A. Shimony, and R. A. Holt, Proposed Experiment to Test Local Hidden-Variable Theories, Phys. Rev. Lett. 23, 880 (1969).

[4] N. Brunner, D. Cavalcanti, S. Pironio, V. Scarani, and S. Wehner, Bell nonlocality, Rev. Mod. Phys. 86, 419 (2014).

[5] A. K. Ekert, Quantum Cryptography Based on Bell's Theorem, Phys. Rev. Lett. 67, 661 (1991).

[6] D. Mayers and A. Yao, Quantum cryptography with imperfect apparatus, in Proceedings 39th Annual Symposium on Foundations of Computer Science, FOCS 1998 (IEEE, Palo Alto, 1998), pp. 503-509.

[7] D. Mayers and A Yao, Self-testing quantum apparatus, Quantum Inf. Comput. 4, 273 (2004).

[8] V. Scarani, The device-independent outlook on quantum physics, Acta Phys. Slovaca 62, 347 (2012).

[9] S. Popescu and D. Rohrlich, Which states violate Bell's inequality maximally? Phys. Lett. A 169, 411 (1992).

[10] S. L. Braunstein and C. M. Caves, Wringing out better Bell inequalities, Ann. Phys. 202, 22 (1990).

[11] R. F. Werner and M. M. Wolf, All-multipartite Bell-correlation inequalities for two dichotomic observables per site, Phys. Rev. A 64, 032112 (2001).

[12] D. Collins, N. Gisin, N. Linden, S. Massar, and S. Popescu, Bell Inequalities for Arbitrarily High-Dimensional Systems, Phys. Rev. Lett. 88, 040404 (2002).

[13] D. Kaszlikowski, L. C. Kwek, J.-L. Chen, M. Żukowski, and C. H. Oh, Clauser-Horne inequality for three-state systems, Phys. Rev. A 65, 032118 (2002).

[14] M. Żukowski and Č. Brukner, Bell's Theorem for General nQubit States, Phys. Rev. Lett. 88, 210401 (2002).

[15] C. Śliwa, Symmetries of the Bell correlation inequalities, Phys. Lett. A 317, 165 (2003).
[16] D. Collins and N. Gisin, A relevant two qubit Bell inequality inequivalent to the CHSH inequality, J. Phys. A: Math. Gen. 37, 1775 (2004).

[17] H. Buhrman and S. Massar, Causality and Tsirelson's bounds, Phys. Rev. A 72, 052103 (2005).

[18] O. Gühne, G. Tóth, P. Hyllus, and H. J. Briegel, Bell Inequalities for Graph States, Phys. Rev. Lett. 95, 120405 (2005).

[19] D. Avis, H. Imai, and T. Ito, On the relationship between convex bodies related to correlation experiments with dichotomic observables, J. Phys. A: Math. Gen. 39, 11283 (2006).

[20] Y.-C. Liang, C.-W. Lim, and D.-L. Deng, Reexamination of a multisetting Bell inequality for qudits, Phys. Rev. A 80, 052116 (2009).

[21] N. Gisin, W. C. Myrvold, and J. Christian, Bell inequalities: Many questions, a few answers, in Quantum Reality, Relativistic Causality, and Closing the Epistemic Circle: Essays in Honour of Abner Shimony (Springer, Dordrecht, 2009), pp. 125-138.

[22] A. Acín, S. Massar, and S. Pironio, Randomness versus Nonlocality and Entanglement, Phys. Rev. Lett. 108, 100402 (2012).

[23] J.-D. Bancal, C. Branciard, N. Brunner, N. Gisin, and Y.-C. Liang, A framework for the study of symmetric full-correlation Bell-like inequalities, J. Phys. A: Math. Theor. 45, 125301 (2012).

[24] B. Grandjean, Y.-C. Liang, J.-D. Bancal, N. Brunner, and N. Gisin, Bell inequalities for three systems and arbitrarily many measurement outcomes, Phys. Rev. A 85, 052113 (2012).

[25] L. Mančinska, Maximally entangled state in pseudo-telepathy games, in Computing with New Resources: Essays Dedicated to Jozef Gruska on the Occasion of His 80th Birthday, edited by C. S. Calude, R. Freivalds, and I. Kazuo (Springer, Cham, 2014), pp. 200-207.

[26] Y.-C. Liang, D. Rosset, J.-D. Bancal, G. Pütz, T. J. Barnea, and N. Gisin, Family of Bell-Like Inequalities as DeviceIndependent Witnesses for Entanglement Depth, Phys. Rev. Lett. 114, 190401 (2015).

[27] S. Schwarz, B. Bessire, A. Stefanov, and Y.-C. Liang, Bipartite Bell inequalities with three ternary-outcome measurements from theory to experiments, New J. Phys. 18, 035001 (2016). 
[28] A. Salavrakos, R. Augusiak, J. Tura, P. Wittek, A. Acín, and S. Pironio, Bell Inequalities Tailored to Maximally Entangled States, Phys. Rev. Lett. 119, 040402 (2017).

[29] E. Oudot, J.-D. Bancal, P. Sekatski, and N. Sangouard, Bipartite nonlocality with a many-body system, arXiv:1810.05636 [New J. Phys (to be published)].

[30] T. Cope and R. Colbeck, Bell inequalities from no-signaling distributions, Phys. Rev. A 100, 022114 (2019).

[31] F. Bacarri, R. Augusiak, I. Šupić, J. Tura, and A. Acín, Scalable Bell inequalities for qubit graph states and robust self-testing, arXiv: 1812.10428.

[32] E. Z. Cruzeiro and N. Gisin, Complete list of tight Bell inequalities for two parties with four binary settings, Phys. Rev. A 99, 022104 (2019).

[33] C. A. Miller and Y. Shi, Optimal Robust Self-Testing by Binary Nonlocal XOR Games, in 8th Conference on the Theory of Quantum Computation, Communication and Cryptography (TQC 2013), Leibniz International Proceedings in Informatics (LIPICs), Vol. 22, edited by S. Severini and F. Brandao (Schloss Dagstuhl-Leibniz-Zentrum fuer Informatik, Dagstuhl, Germany, 2013), pp. 254-262.

[34] T. H. Yang and M. Navascués, Robust self-testing of unknown quantum systems into any entangled two-qubit states, Phys. Rev. A 87, 050102(R) (2013).

[35] C. Bamps and S. Pironio, Sum-of-squares decompositions for a family of Clauser-Horne-Shimony-Holt-like inequalities and their application to self-testing, Phys. Rev. A 91, 052111 (2015).

[36] I. Šupić, R. Augusiak, A. Salavrakos, and A. Acín, Self-testing protocols based on the chained Bell inequalities, New J. Phys. 18, 035013 (2016).

[37] J. Kaniewski, Analytic and Nearly Optimal Self-Testing Bounds for the Clauser-Horne-Shimony-Holt and Mermin Inequalities, Phys. Rev. Lett. 117, 070402 (2016).

[38] O. Andersson, P. Badziagg, I. Bengtsson, I. Dumitru, and A. Cabello, Self-testing properties of gisin's elegant Bell inequality, Phys. Rev. A 96, 032119 (2017).

[39] J. Kaniewski, I. Šupić, J. Tura, F. Bacarri, A. Salavrakos, and A. Acín, Maximal nonlocality from maximal entanglement and mutually unbiased bases, and self-testing of two-qutrit quantum systems, arXiv:1807.03332 [Quantum (to be published)].

[40] I. Šupić and J. Bowles, Self-testing of quantum systems: A review, arXiv:1904.10042.

[41] S. Pironio, Lifting Bell inequalities, J. Math. Phys. 46, 062112 (2005).

[42] D. Rosset, J.-D. Bancal, and N. Gisin, Classifying 50 years of Bell inequalities, J. Phys. A: Math. Theor. 47, 424022 (2014).

[43] J. Barrett, N. Linden, S. Massar, S. Pironio, S. Popescu, and D. Roberts, Nonlocal correlations as an information-theoretic resource, Phys. Rev. A 71, 022101 (2005).

[44] K. T. Goh, J. Kaniewski, E. Wolfe, T. Vertesi, X. Wu, Yu Cai, Y.-C. Liang, and V. Scarani, Geometry of the set of quantum correlations, Phys. Rev. A 97, 022104 (2018).

[45] P.-S. Lin, D. Rosset, Y. Zhang, J.-D. Bancal, and Y.-C. Liang, Device-independent point estimation from finite data and its application to device-independent property estimation, Phys. Rev. A 97, 032309 (2018).
[46] S. Popescu and D. Rohrlich, Quantum nonlocality as an axiom, Found. Phys. 24, 379 (1994).

[47] A. Coladangelo, K. T. Goh, and V. Scarani, All pure bipartite entangled states can be self-tested, Nat. Commun. 8, 15485 (2017).

[48] T. Coopmans, J. Kaniewski, and C. Schaffner, Robust self-testing of two-qubit states, Phys. Rev. A 99, 052123 (2019).

[49] M. McKague, T. H. Yang, and V. Scarani, Robust self-testing of the singlet, J. Phys. A: Math. Theor. 45, 455304 (2012).

[50] K. F. Pál, T. Vértesi, and M. Navascués, Device-independent tomography of multipartite quantum states, Phys. Rev. A 90, 042340 (2014).

[51] A. Natarajan and T. Vidick, A quantum linearity test for robustly verifying entanglement, in Proceedings of the 49th Annual ACM SIGACT Symposium on Theory of Computing, STOC 2017 (ACM, New York, 2017), pp. 1003-1015.

[52] A. Coladangelo, Generalization of the Clauser-HorneShimony-Holt Inequality Self-Testing Maximally Entangled States of Any Local Dimension, Phys. Rev. A 98, 052115 (2018).

[53] P. Shadbolt, T. Vértesi, Y.-C. Liang, C. Branciard, N. Brunner, and J. L. O'Brien, Guaranteed violation of a Bell inequality without aligned reference frames or calibrated devices, Sci. Rep. 2, 470 (2012).

[54] C. Branciard, Detection loophole in Bell experiments: How postselection modifies the requirements to observe nonlocality, Phys. Rev. A 83, 032123 (2011).

[55] J. I. de Vicente, On nonlocality as a resource theory and nonlocality measures, J. Phys. A: Math. Theor. 47, 424017 (2014)

[56] T. H. Yang, T. Vértesi, J.-D. Bancal, V. Scarani, and M. Navascués, Robust and Versatile Black-Box Certification of Quantum Devices, Phys. Rev. Lett. 113, 040401 (2014).

[57] J.-D. Bancal, S. Pironio, A. Acín, Y.-C. Liang, V. Scarani, and N. Gisin, Quantum non-locality based on finite-speed causal influences leads to superluminal signalling, Nat. Phys. 8, 867 (2012).

[58] T. J. Barnea, J.-D. Bancal, Y.-C. Liang, and N. Gisin, Tripartite quantum state violating the hidden-influence constraints, Phys. Rev. A 88, 022123 (2013).

[59] F. J. Curchod, N. Gisin, and Y.-C. Liang, Quantifying multipartite nonlocality via the size of the resource, Phys. Rev. A 91, 012121 (2015).

[60] A. S. Sørensen and K. Mølmer, Entanglement and Extreme Spin Squeezing, Phys. Rev. Lett. 86, 4431 (2001).

[61] D. Rosset, Ä. Baumeler, J.-D. Bancal, N. Gisin, A. Martin, M.-O. Renou, and E. Wolfe, Algebraic and geometric properties of local transformations (unpublished).

[62] J. Kaniewski, A weak form of self-testing, arXiv:1910.00706 (2019).

[63] M. Navascués, S. Pironio, and A. Acín, Bounding the Set of Quantum Correlations, Phys. Rev. Lett. 98, 010401 (2007).

[64] T. Moroder, J.-D. Bancal, Y.-C. Liang, M. Hofmann, and O. Gühne, Device-Independent Entanglement Quantification and Related Applications, Phys. Rev. Lett. 111, 030501 (2013). 